\title{
Marginal corneal abscess associated with adult chlamydial ophthalmia
}

\author{
S DAROUGAR AND N D VISWALINGAM
}

From the Section of Virology, Institute of Ophthalmology, Judd Street, London WC1H 9QS, and the External Eye Diseases Clinic, Moorfields Eye Hospital, City Road, London EC1V2PD

SUMMARY In four patients with an adult chlamydial ophthalmia small, marginal corneal abscesses were detected. These corneal abscesses were associated with unilateral papillary and follicular conjunctivitis and punctate keratitis. In these patients no bacteria was isolated from the abscesses, but Chlamydia trachomatis was isolated from materials collected from the abscesses and from the conjunctival swabbings. In addition all patients had microbiologically proved concomitant chlamydial genital infections. The clinical signs resolved after topical treatment with rifampicin or tetracycline eye ointment for six weeks or systemic treatment with tetracycline for two weeks. Because of concomitant chlamydial genital infection it is advisable to treat patients with adult chlamydial ophthalmia with systemic tetracycline and to refer these patients and their consorts for investigation and treatment of their genital infection.

Adult chlamydial ophthalmia (ACO) of sexually transmitted origin has been reported with increasing frequency. ${ }^{1}$ The infection is commonly associated with papillary and follicular responses in the palpebral conjunctiva, fine or coarse epithelial and subepithelial punctate keratitis. ${ }^{23}$ In this report we present four cases of corneal abscess which developed in association with chlamydial conjunctivitis and in which Chlamydia trachomatis was isolated from the abscess material.

\section{Material and methods}

\section{CLINICAL EXAMINATION}

The patients were referred to the External Eye Diseases Clinic, Moorfields Eye Hospital, London. They were examined by a Haag-Streit slit-lamp, and the severity of symptoms and signs was graded and recorded as described previously. ${ }^{4}$

\section{LABORATORY INVESTIGATIONS}

Chlamydial culture. Conjunctival swabbings were collected from all areas of palperbral conjunctiva. ${ }^{5}$ Swabbings were also collected from the corneal abscess after the cornea had been washed several times and the epithelium over the abscess removed.

Correspondence to Professor S Darougar, Section of Virology, Institute of Ophthalmology, Judd Street, London WC1H 9QS.
Each swab was placed in a plastic vial containing 2SP transport medium ${ }^{6}$ with additional fetal bovine serum $3 \% \mathrm{v} / \mathrm{v}$. The methods of transport and storage of the specimens and methods of culture in irradiated McCoy cells or cycloheximide treated McCoy cells are described elsewhere. ${ }^{78}$

Bacterial culture. Swabs for bacterial cultures were collected from the lower fornix of the palpebral conjunctiva, the lid margins, and the corneal abscess separately and plated on blood agar for bacterial culture.

Antibody detection. Blood samples were collected by venepuncture or by sponges following a finger prick. ${ }^{9}$. These were tested by a modified microimmunofluorescence test ${ }^{10}$ for the presence and levels of type-specific antichlamydial antibodies.

\section{Results}

CASE 1

A 26-year-old married woman presented with a seven-month history of a slightly red and irritable right eye, with a sticky yellowish discharge. During this period she had been treated with chloramphenicol drops $0.5 \%$ or neomycin drops $0.5 \%$, and more latterly with prednisolone drops $0.3 \%$, all of which gave temporary relief. For five days she experienced an increasing gritty sensation and severe photophobia. 
The visual acuity in the right eye was $6 / 12$ and in the left $6 / 6$. In the right eye there was a slight ptosis of the upper lid, and the bulbar conjunctiva was mildly chemotic and hyperaemic. The palpebral conjunctiva showed a moderate papillary and follicular hyperplasia. At the limbus an active limbitis with a $1 \mathrm{~mm}$ pannus and associated marginal diffuse infiltration were present. The cornea showed some coarse epithelial and subepithelial punctate keratitis near the limbus. At the 6 o'clock position there was a well circumscribed corneal abscess measuring $2 \mathrm{~mm} \times$ $3 \mathrm{~mm}$ involving the epithelium to mid stroma, with a mild stromal oedema surrounding the abscess. No other abnormality was detected in the anterior or posterior segment of the eye. The cornea of the left eye was clear, while the palpebral conjunctiva showed a mild papillary and follicular reaction. Bacterial culture of the abscess material and conjunctiva was negative, whereas a few colonies of Staphylococcus epidermidis were isolated from the lid margins. $C$. trachomatis was isolated from the corneal abscess and conjunctiva. Serum IgG antibody against $C$. trachomatis serotypes $\mathrm{D}$ to $\mathrm{K}$ was present at a level of $1 / 256$. Subsequent genital examination showed a gross cervicitis and a positive chlamydial culture.

The patient recovered fully after two weeks of oral doxycycline with an initial dose of $300 \mathrm{mg}$ followed by $100 \mathrm{mg}$ daily. There was no recurrence of infection during the six months' follow-up.

\section{CASE 2}

A man 48 years old had a six-week history of progressive redness, a mucopurulent discharge, and photophobia of the right eye.

The visual acuity in the right eye was $6 / 9$ and in the left eye $6 / 6$. The bulbar conjunctiva of the right eye was mildly hyperaemic, while the palpebral conjunctiva showed severe papillary and follicular hyperplasia mainly in the fornices. The right cornea showed epithelial erosions, and fine and coarse epithelial punctate keratitis mainly in the upper half. The left eye was normal. He was treated with rifampicin eye ointment $1 \%$ three times daily for six weeks, with good resolution of clinical signs and symptoms.

Two weeks after completion of the above treatment he returned to the clinic complaining of redness and intense photophobia in the right eye. The bulbar conjunctiva of the eye was moderately hyperaemic, while the palpebral conjunctiva showed a fairly moderate papillary and follicular reaction. There was a little scattered epithelial punctate keratitis mainly in the upper half of the cornea. At the 10 o'clock position a well circumscribed corneal abscess measuring $2.5 \mathrm{~mm} \times 1.5 \mathrm{~mm}$ was present. A mild

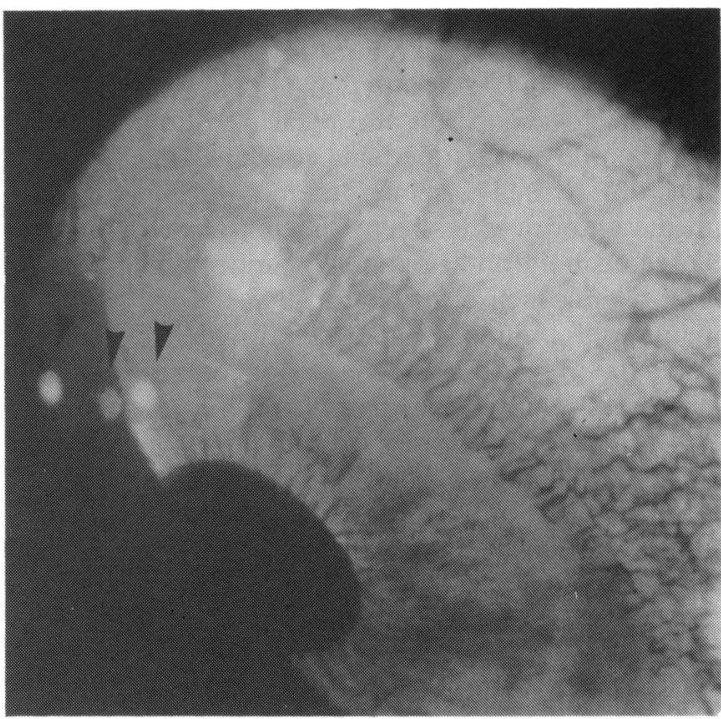

Fig. 1 Marginal corneal abscesses (arrowed) associated with adult chlamydial ophthalmia (case 3).

stromal oedema surrounded the abscess, with a faint flare in the aqueous. No other abnormality was detected in the anterior and posterior segments of the eye. The left eye was normal. Bacterial culture of the abscess material and conjunctiva was negative, but a few colonies of Staphylococcus epidermidis was isolated from the lid margins. $C$. trachomatis was isolated from the corneal abscess and conjunctiva. Serological test showed the presence of IgG antibody against $C$. trachomatis serotypes $\mathrm{D}$ to $\mathrm{K}$ at a level $1 / 32$.

The patient was treated with achromycin eye ointment three times daily for six weeks. Subsequently he was treated with oral tetracycline for two weeks because of a chlamydial genital infection. The patient recovered fully after treatment, and there was no recurrence of infection during the one-year follow-up.

\section{CASE 3}

A man 28 years old presented with a three-week history of a red, sticky, and irritable right eye.

The visual acuity was $6 / 12$ in the right eye and $6 / 6$ in the left eye. The lids of the right eye were swollen, there was a tender preauricular and submandibular lymphadenopathy, a mucopurulent discharge, and severely hyperaemic bulbar conjunctiva. The palpebral conjunctiva showed a severe papillary and follicular hyperplasia. The follicles were predominantly in the upper and lower fornices. A few follicles were also present on the plica and caruncle. The limbus was oedematous, with marginal diffuse infil- 
tration and a micropannus. In the cornea three small but discrete and well circumscribed abscesses close to the limbus were present (Fig. 1). The largest was at the 3 o'clock position and measured $2 \mathrm{~mm} \times 3 \mathrm{~mm}$, with a mild stromal oedema round it. There were a few folds in the Descemet's membrane and mild flare in the anterior chamber. No other abnormality was detected in the anterior and posterior segments of the eye. The cornea of the left eye was clear, while the palpebral conjunctiva showed a mild papillary and follicular hyperplasia. Bacterial cultures of the abscesses and conjunctiva were all negative. $C$. trachomatis was isolated from the corneal abscesses and conjunctiva. Serum IgG antibody against $C$. trachomatis $\mathrm{D}$ to $\mathrm{K}$ was detected at a level of $1 / 128$.

The patient was treated with rifampicin eye ointment three times daily for six weeks, which was supplemented with oral tetracycline for two weeks because of a chlamydial genital infection. The patient recovered fully after treatment, and there was no recurrence of infection during the one-year followup.

\section{CASE 4}

A man 23 years old attended the clinic with a 10-day history of a red, painful, photophobic, and sticky right eye. He also complained of increasing aching in the right ear.

The visual acuity in the right eye was $6 / 9$ and in the left eye $6 / 5$. The right upper lid showed slight ptosis, and the bulbar conjunctiva was moderately hyperaemic. A non-tender right preauricular and submandibular lymphadenopathy was present. The right cornea showed a micropannus, some marginal punctate keratitis, and at the 10 o'clock position a well circumscribed abscess slightly away from the limbus measuring $2 \mathrm{~mm} \times 2 \mathrm{~mm}$ and surrounded by a very mild stromal oedema. The palpebral conjunctiva showed severe papillary and follicular responses. No other abnormality was detected in the right eye. In the left eye, apart from a mild papillary hyperplasia of the upper tarsal conjunctiva, no other abnormality was present. Bacterial culture of the abscess material was negative, but a few colonies of Staphylococcus epidermidis and Bacillus xerosis were isolated from the conjunctiva and lid margins. $C$. trachomatis was isolated from the corneal abscess and conjunctiva. Serum IgG antibody against $C$. trachomatis serotypes $\mathrm{D}$ to $\mathrm{K}$ was detected at a level of $1 / 256$.

The patient was treated with rifampicin eye ointment three times daily for six weeks. He was subsequently treated with oral tetracycline because of a chlamydial genital infection. He recovered fully after treatment, and there was no recurrence of infection during the one-year follow-up.

\section{Discussion}

The four cases of ACO reported had developed small marginal abscesses which were associated with a unilateral papillary and follicular conjunctivitis and punctate keratitis. The people specially at risk of developing a corneal ulcer and/or abscess are contact lens wearers, those who have had corneal surgery or trauma or corneal surface disorders, and those with chronic bacterial conjunctivitis. ${ }^{11}$ In these cases the corneal abscess tends to be large, and it is generally associated with severe blepharitis, or a contact lens which is not sterilised routinely, or trauma, or corneal surface abnormalities associated with abnormal tear function. Our patients did not belong to any of these risk groups. Bacterial cultures from their corneal abscesses were all negative. $C$. trachomatis was isolated from the abscess material, and all the patients had microbiologically proved concomitant chlamydial ocular and genital infections. It may be argued that the isolation of $C$. trachomatis from the abscess material may have been due to contamination of the corneal specimen with tears containing infectious chlamydial particles shed from the conjunctiva. In order to avoid such contamination we kept the lids apart to prevent blinking, washed the cornea several times, and ruptured the abscess membrane prior to collection of the specimen from the abscess.

The corneal abscesses which developed in our patients were rather small; hence clinically they might be mistaken for subepithelial punctate keratitis (SEPK) or marginal abscess associated with blepharoconjunctivitis. Generally SEPK presents as dull yellow and small opacities (less than $1 \mathrm{~mm}$ in diameter) located in the subepithelial and superficial stroma and with no stromal oedema. ${ }^{3}$ In contrast the corneal abscesses detected in our patients were dense white lesions located more deeply in the stroma and were surrounded with stromal oedema. The clinical differential diagnosis of chlamydial corneal abscess from bacterial marginal abscess is not feasible; hence it is necessary to rely on chlamydial and bacterial diagnostic tests.

In our patients the corneal abscesses and conjunctivitis responded well to both topical and systemic antichlamydial treatment. However, because of concomitant genital infection we recommend systemic treatment with tetracycline and referral of patients and their consorts for investigation of chlamydial genital infection and necessary treatment.

\section{References}

1 Annual report of the Chief Medical Officer of the Department of Health and Social Security for the year 1983. Sexually transmitted diseases. Br J Vener Dis 1985; 61: 104-7. 
2 Viswalingam ND, Wishart MS, Woodland RM. Adult chlamydial ophthalmia. Br Med Bull 1983; 39: 117-22.

3 Jones BR. Ocular syndromes of TRIC virus infection and their possible genital significance. Br J Vener Dis 1964; 40: 4-15.

4 Darougar S, Viswalingam ND, Treharne JD, Kinnison JR, Jones BR. Treatment of TRIC infection of the eye with rifampicin or chloramphenicol eye ointment. $\mathrm{Br} J$ Ophthalmol 1977; 61: 255-9.

5 Darougar S, Jones BR. Conjunctival swabbing for isolation of TRIC agent. Br J Ophthalmol 1971; 55: 585-90.

6 Gordon FB, Harper IA, Quan AL, Treharne JD, Dwyer RStC, Garland JA. Detection of Chlamydia (Bedsonia) in certain infections of man. I. Laboratory procedures: Comparison of yolk sac and cell culture for detection and isolation. J Infect Dis 1969; 120: 451-62.

7 Darougar S, Kinnison JR, Jones BR. Simplified irradiated McCoy cell culture for isolation of chlamydiae. In: Nichols RL, ed. Trachoma and related disorders caused by chlamydial agents. Amsterdam: Excerpta Medica, 1970: 63-70.

8 Ripa KT, Mardh PA. New simplified culture techniques for Chlamydia trachomatis. In: Hobson D, Holmes KK, eds. Nongonococcal urethritis and related infections. Washington, DC: American Society for Microbiology, 1977: 323-7.

9 Darougar S, Treharne JD, Minassian D, El-Sheikh H, Dines RJ, Jones BR. Rapid serological test for diagnosis of chlamydial ocular infections. Br J Ophthalmol 1978; 62: 503-8.

10 Treharne JD, Darougar S, Jones BR. Modification of the microimmunofluorescence test to provide a routine serodiagnostic test for chlamydial infection. J Clin Pathol 1977; 30: 510-7.

11 Wilhelmus KR. Bacterial corneal ulcers. In: Randall J Olsen, eds. Int Ophthalmol Clin 1984; 24: 1-16.

Accepted for publication 17 August 1987. 